\title{
Prácticas JUVENILES RURALES E INDÍGENAS DE PRODUCCIÓN SIMBÓLICA. El CASO DE Santa María AteXcac, Huejotzingo, Puebla
}

\section{Rural and Indigenous Youth Practices of Symbolic Production. The Case of Santa María Atexcac, Huejotzingo, Puebla}

\author{
Noel Pérez-Vargas* \\ José Arturo Méndez-Espinoza** \\ DOI: https://doi.org/10.29043/liminar.vl8i2.765
}

Resumen: El objetivo principal del texto es comprender el sentido de las prácticas juveniles de las bandas y bailes sonideros de Santa María Atexcac, Puebla, y conocer los factores que inciden en su aparición, así como los intereses que se persiguen con su continuidad. La investigación se basó en el método etnográfico. Se concluye que el sentido de las bandas y bailes sonideros deriva de las tensiones territoriales entre jóvenes y adultos, y que uno de los principales intereses juveniles que generan tal tensión está asociado con la búsqueda de un lugar en el espacio social de Atexcac.

Palabras clave: jóvenes indígenas, prácticas simbólicas, espacio social, transformaciones territoriales.

Abstract: The main objective of this ethnographic research is to understand the meaning behind young people's participation in the bands and dances of Santa María Atexcac, Puebla, and appreciate the factors that influenced their emergence and what interests are pursued therein. We found that the meaning of the bands and dances has to do with territorial tensions that exist between young people and adults. In addition, one main interest of young people that generates tension involves their search for a place within the social space of Atexcac.

Keywords: indigenous youth, symbolic practices, social space, territorial transformations.

\footnotetext{
* Noel Pérez Vargas. Maestro en Ciencias en Estrategias para el Desarrollo Agrícola Regional por el Colegio de Postgraduados, Campus Puebla, México. Temas de especialización: juventud, desigualdad social y procesos rurales. Correo electrónico: noel.pe.va@gmail.com. ORCID: https://orcid.org/0000-0002-5679-9451

** José Arturo Méndez Espinoza. Doctor en Planificación Territorial y Desarrollo Regional por la Universidad de Barcelona, España. Profesorinvestigador titular del Colegio de Postgraduados, Campus Puebla, México. Temas de especialización: configuraciones territoriales y
}

estrategias de reproducción social de la agricultura familiar en espacios rurales y periurbanos. Correo electrónico: jamendez@colpos.mx. Autor de correspondencia. ORCID: http://orcid.org/0000-0002-9733-4175

Enviado a dictamen: 8 de abril de 2019. Aprobación: 11 de novienbre de 2019.

Revisiones: 2. 


\section{Introducción}

$\longrightarrow$ on el interés de contribuir a la comprensión del sector juvenil en las sociedades rurales e indígenas de México, en este texto se analizan las prácticas de los jóvenes de una sociedad indígena en un contexto rural del centro del país, particularmente los bailes sonideros y las bandas como prácticas de producción simbólicas:

Si bien la temática indígena ha sido recurrente y tradicional en la antropología mexicana, el tema de los jóvenes indígenas no ha sido central en la investigación antropológica ni social; por lo menos así lo señala una somera revisión de la literatura etnográfica sobre los pueblos indios de México producida entre los años cincuenta y mediados de los noventa (Urteaga, 2008:670).

En la actualidad, los estudios sobre la juventud indígena concuerdan en que se trata de un sector poco estudiado, por lo que se ha llegado a considerar como una nueva condición etaria. Esta visión destaca que entre los factores que han visibilizado esta categoría se encuentran "la migración, la introducción de la escuela secundaria y los medios de comunicación, aunque la migración es determinante en este proceso" (Urteaga, 2008:678)

El análisis de las prácticas juveniles revisadas en este texto se pretende que contribuya a la comprensión del fenómeno juvenil en las sociedades contemporáneas de origen indígena. Particularmente, permite visualizar la manera en que factores como la migración, la escolarización y los medios de comunicación influyen en la construcción de las prácticas juveniles, pero, además, se reconoce la influencia que ejerce la herencia cultural indígena en tal construcción. Así, se considera que: "Esta juventud es heredera de todo el acervo cultural de sus pueblos y al mismo tiempo selecciona aquellos elementos que le permiten dialogar con la sociedad nacional, readecuando y cotejando los papeles que como sociedad indígena les ha asignado un marco poscolonial de referencia" (García, 2012:77).
En la comunidad de Santa María Atexcac, los bailes sonideros son celebraciones nocturnas predominantemente juveniles que tienen lugar en espacios privados y públicos de la localidad. El festejo de los bailes se asocia a cumpleaños de jóvenes, a festividades de la localidad y, principalmente, al aniversario de la fundación de las bandas juveniles del lugar.

El sonidero mexicano es un espectáculo que consiste en la colocación de grandes equipos de audio e iluminación en el espacio público. En un sonidero la gente se reúne a bailar. Dicho espectáculo es dirigido por una persona, algo así como un maestro de ceremonias, que desempeña dos funciones:

a) Colocar música tropical grabada ya sea en discos de vinilo o a través de una computadora en una tornamesa.

b) Amenizar el evento a través de un micrófono, mismo que utiliza para enviar saludos a los asistentes, invitar a que éstos bailen y presentar las canciones que están sonando en la tornamesa (Salazar, 2018:25).

El fenómeno de las bandas emergió a principios de la primera década del siglo XXI. Las bandas son grupos de jóvenes de entre 13 y 20 años de edad conformados principalmente por hombres, aunque el número de mujeres que se enrolan va en aumento. Las bandas se ubican en diferentes puntos de la localidad y rivalizan simbólica y físicamente de acuerdo con la división territorial tradicional que distingue al barrio de arriba del barrio de abajo; en este sentido, cada banda tiene su territorio. Entre sus actividades principales se encuentran las reuniones diurnas y nocturnas en el espacio público y su participación en los bailes sonideros.

Santa María Atexcac se localiza en el municipio de Huejotzingo, Puebla, en los límites del volcán Iztaccíhuatl. En 2010 la población total era de 2978 habitantes (INEGI, 2010), de la cual 538 personas tenían entre 15 y 24 años de edad. Entre las actividades económicas que predominan se encuentran la agricultura y la migración hacia la Ciudad de México y 
hacia Filadelfia, estaúltima en Estados Unidos; además, se presenta movilidad laboral a ciudades de la región como Puebla, San Martín Texmelucan y Huejotzingo, donde se emplean principalmente en el sector de la construcción como albañiles.

El objetivo que orienta el documento es comprender el sentido de las prácticas del subcampo que concentra bailes sonideros y bandas de Santa María Atexcac. La hipótesis de trabajo fue que estas prácticas surgen de las tensiones territoriales entre jóvenes y adultos derivadas de las dinámicas migratorias, y son readaptadas de acuerdo con las particularidades del lugar, en donde es central la necesidad de la población juvenil de ocupar un lugar en el espacio social.

Para analizar los fenómenos juveniles de los bailes sonideros y las bandas se recurre al aparato teórico metodológico de Pierre Bourdieu (2011), quien afirma que las prácticas forman parte de un campo de posiciones determinadas por el volumen de capital. En este campo, los agentes sociales que están interesados en el juego buscan hacerse de un bien denominado capital.

\begin{abstract}
En su aprehensión sincrónica, los campos se presentan como "sistemas de posiciones y de relaciones entre posiciones" [...] se define entre, otras cosas, definiendo lo que está en juego [...] La estructura de un campo es un estado — en el sentido de momento histórico- de la distribución de un momento dado del tiempo, del capital específico que allí está en juego. [...] puede decirse también que su estructura es un estado de las relaciones de fuerza entre los agentes y las instituciones comprometidos en el juego. [...] Además de un campo de fuerzas, un campo social determinado constituye un campo de luchas destinadas a conservar o transformar ese campo de fuerzas (Gutiérrez, 2005:31-32).
\end{abstract}

De esta manera, el análisis de las prácticas de los jóvenes de Atexcac interesados en los bailes sonideros y las bandas parte del entendido de que ellos tienen un interés en común, que es obtener el bien del reconocimiento o capital simbólico. Los jóvenes ocupan diferentes posiciones de acuerdo con su volumen de capital, es decir, de acuerdo con sus recursos, con los que tienden a ocupar distintas posiciones, lo que les facilita o limita el acceso al capital simbólico o reconocimiento.

El interés que muestran los jóvenes en Atexcac hacia las prácticas en cuestión se comprende como parte del habitus, en tanto estructura interna que vincula al agente social con las prácticas. El habitus se expresa de manera individual y colectiva; en la primera forma el "principio de las diferencias entre los habitus individuales reside en la singularidad de las trayectorias sociales" (Bourdieu, 2013:98).

Las prácticas de los bailes sonideros y de las bandas de Atexcac se comprenden como parte de un campo, debido a que el capital simbólico no es exclusivo de estas prácticas, es decir, se puede obtener por otros medios; por ejemplo, los jóvenes que forman parte de una familia que tienen un representante encargado de una mayordomía, se hacen visibles en la comunidad y adquieren cierto reconocimientos social.

Cada subcampo, en este caso, está intrínsecamente relacionado con el espacio social de los jóvenes en Atexcac. Las prácticas del baile sonidero y de las bandas son reproducidas por una fracción de estos jóvenes, y tienen sentido en el espacio social de la localidad. "La estructura de un campo es un estado - en el sentido de momento histórico- de la distribución, en un momento dado del tiempo, del capital específico que allí está en juego" (Bourdieu, 2015:12). El campo también tiene un sentido histórico, puesto que el capital que se disputa en un tiempo determinado: "ha sido acumulado en el curso de las luchas anteriores, que orienta las estrategia de los agentes que están comprometidos en el campo y que puede cobrar diferentes formas, no necesariamente económicas, como el capital social, el cultural, el simbólico, y cada una de sus subespecies" (Bourdieu, 2015:12).

Conestabase, dosejesguían la presenteinvestigación. Por un lado, la construcción histórica del campo, que es la historia de las prácticas, y prioritariamente la historia de la constitución de los subcampos juveniles, que permite comprender los factores que contribuyeron al surgimiento de las prácticas de estudio. Por otro lado, el análisis de los subcampos, que permite reconocer la 
trascendencia de los bailes sonideros y bandas para los jóvenes contemporáneos de Atexcac, y que contribuye a comprender el sentido de las prácticas de la generación juvenil contemporánea a través del análisis de los intereses, las acciones y las posiciones juveniles respecto a las de los adultos.

Bajo este esquema, la juventud se analiza como una clase sociocultural que forma parte de un espacio social y que es producto de relaciones sociohistóricas. Este esquema contribuye a la interpretación de la juventud rural indígena contemporánea y abona a la discusión sobre el papel de los jóvenes tras una serie de trasformaciones estructurales de la ruralidad en México.

La investigación es de tipo cualitativo. Para la recolección de la información se hicieron dos estancias de campo en las que se usaron instrumentos de tipo etnográfico como la observación directa y participante. Además, se hicieron dos grupos focales con jóvenes de la comunidad y veinte entrevistas, la mitad de ellas de tipo informal y la otra mitad de tipo semiestructurado. El criterio de recolección de datos fue el de saturación teórica, que se basa en la obtención de información hasta que esta se hace repetitiva o ya no aporta nada nuevo a los intereses que persigue la investigación (Hernández, 2014).

A las citas textuales de los informantes se les asignó una clave; particularmente en este texto se usan infa- $x$ para referirse a los informantes mayores de 50 años, y grupo focal- $x$ cuando se cita a uno de los grupos focales que se desarrolló en la institución escolar de nivel medio superior de la localidad. En las citas de los grupos focales se distingue la opinión entre hombres $(\mathrm{H})$ y mujeres (M).

\section{Historia del campo: transición a la juventud contemporánea}

Es factible afirmar, a partir del estudio realizado, que uno de los factores que contribuyó al surgimiento de las prácticas juveniles simbólicas de análisis fue la movilidad laboral, la cual en Santa María Atexcac presenta dos momentos importantes. El primero se generó hacia mediados de la década de 1960 y detonó en la década de 1980, momento en que la agricultura y la tala de árboles dejaron de soportar la economía familiar del lugar, y el segundo a mediados de la década de 1990 con destino a Filadelfia, Estados Unidos. Los que ahora son adultos mayores de 60 años fueron pioneros en llevar sus productos a la hoy Ciudad de México.

Pues, le digo que en 1960, en esos años, ya empezamos a ir a vender. No, pues, desde la edad de unos 18 años. Yo anteriormente, aquí, en el pueblo, íbamos a trabajar mucho la madera. Cortaba uno madera a pura sierra voladora, que no sé si las conoce, que uno arriba y otro abajo, se habría un agujero [...] y se atravesaban unos palos. Después, cuando yo ya tuve mi esposa, pues ya no tanto me gustó eso, ya me dediqué al comercio. Pues mire, primero aquí lo bajaba uno con animales a Huejotzingo, y ahí siempre ha habido carros que llevaban la carga para México. En temporada de fruta venía un carro a cargar, y aunque los caminos estaban muy mal... pero venía un carro a cargar y así se llevaba para México (infa-2, agosto de 2017).

Los productos que se comerciaban eran principalmente la pera y la manzana. Y una de las principales razones por las que comenzaron a ir a vender a la Ciudad de México fue la de superar la intermediación comercial que realizaba la gente de la cabecera municipal, Huejotzingo.

Esta generación se caracterizó por su temprana inserción al sector laboral debido, entre otros factores, a su arraigo a la cultura campesina. En este texto, el concepto de generación alude a "referentes simbólicos que identifican vagamente a los agentes socializados en unas mismas coordenadas temporales" (Feixa, Férnandez y Figueras, 2016:113). Así, los jefes de familia de ese momento histórico inducían a sus hijos a la pronta inserción en la reproducción de la unidad doméstica, lo que implicaba acercarlos desde temprana edad al sector laboral. Por su parte, las mujeres se perfilaban hacia su rol particular en la dinámica campesina, pero también eran orientadas hacia las labores del hogar y la crianza de los hijos. 
Por lo anterior, la juventud en Atexcac del periodo que va de 1960 a 1980 se comprende distante de la cultura juvenil que emergía en las sociedades urbanas metropolitanas. En ese momento una pequeña fracción de los jóvenes de Atexcac dilataba sus compromisos nupciales y tuvo acceso a otros escenarios sociales por efectos de la movilidad laboral. Sin embargo, esta situación estuvo presente solo entre los hombres. Además, no se relaciona con las dinámicas de los campos juveniles que se analizan en este texto, aunque sí contribuyó a su formación por efectos de la movilidad laboral.

Hacia principios de la década de los ochenta se consolidó la red mercantil entre Atexcac y la Ciudad de México y se comenzaron a diversificar las ocupaciones laborales de los migrantes del lugar.

Aquí, primeramente, pues fue una señora de no sé de qué parte que vive allá en México, y que mandó a ocupar gente de aquí, inclusive una de mis hijas fue. Estuvo por allá vendiendo gorditas, y empezaron a ver las gentes y empezaron a ir, y ya empezaron a vender. Aquí muchos muchachos también iban a vender refresco, y se preparaban los refrescos con limón y Tehuacán [agua mineral]. Hacían los preparados. Se vendían también los ostiones, hubo un tiempo en que muchos muchachos se fueron a vender ostiones (infa-2, agosto de 2017).

En ese momento histórico la población comenzó a asentarse en el lugar de destino, e incluso algunas familias de Atexcac tuvieron acceso a viviendas en la Ciudad de México debido al terremoto de 1985. Con la oportunidad de asentamiento en la ciudad, los pobladores de Atexcac comenzaron a establecerse en diversos lugares de la capital y en municipios conurbados del Estado de México. En tal sentido, un informante comentó:

Más o menos tenía 11 años cuando me fui para allá. Incluso ahí ya estudié la secundaria. El año, no recuerdo exactamente en qué año [...] me fui con mis hermanas, ellas estaban allá, y todavía no llevaba una idea de cómo o qué quería. Estuve ahí, estudié la secundaria, ahí trabajaba también, después yo ya [...] estuve en el ejército también, me metí al ejército a los 16 años, salí de ahí y me fui para Estados Unidos (infa-1, septiembre de 2016).

Las generaciones de jóvenes de principios de la década de 1980 a mediados de 1990, en particular aquellos con redes en el entonces Distrito Federal, se sumaron a nuevas dinámicas sociales específicas, particularmente a la migración a la capital del país y hacia Estados Unidos. Con esto emergieron nuevos intereses vinculados a las dinámicas socioculturales del destino laboral, entre los que destaca un incremento en el interés por el capital económico. Sin embargo, los jóvenes de esta generación no variaron su empatía hacia la dinámica sociocultural dominante de la generación antecesora, principalmente hacia el sistema de organización comunitario.

Así, esta generación todavía reconocía y privilegiaba la inserción temprana a la dinámica laboral; reproducía la tradición de contraer compromisos nupciales a temprana edad - a partir de los 12 años-; estaba comprometida y cumplía con las obligaciones sociales comunitarias, y regularmente truncaba las trayectorias vinculadas a la escolaridad entre los 12 y los 14 años.

Si bien estas generaciones juveniles entraron en contacto directo con las dinámicas socioculturales de los jóvenes de la Ciudad de México y comenzaron a apropiarse de gustos juveniles como la música, los bailes sonideros y la vestimenta, no presenciaron la aparición del sistema simbólico de prácticas juveniles de Atexcac. Es importante reconocer que en esta generación las mujeres jóvenes se integraron activamente a la dinámica migratoria a través de las redes familiares, y también fueron partícipes de la adopción e inserción de nuevos estilos juveniles en Atexcac.

La dinámica sociocultural juvenil contemporánea de esta localidad se distingue por su enrolamiento en diversas prácticas, que van desde la reproducción de los deberes familiares y comunitarios a las dinámicas exclusivamente juveniles. En el primer caso se distinguen los deberes en la familia y los religiosos. En 
general, la juventud contribuye completa o parcialmente a las actividades económicas - principalmente agrícolas - de acuerdo con su disponibilidad de tiempo, que está marcada por la continuidad o el abandono de la trayectoria escolar. Con el mismo criterio de escolaridad se integran las prácticas de las "faenas", que es un sistema de préstamo de mano de obra que contribuye al mantenimiento material de la localidad, por ejemplo, a mitigar los daños que la lluvia ocasiona a las vías de comunicación y a las tuberías de agua potable.

En el caso de las prácticas religiosas, los jóvenes pueden llevar a cabo diversas actividades que forman parte de los sistemas religiosos locales: evangélico, mormón y católico. En cada religión es visible la distinción de género de los roles entre hombres y mujeres; por ejemplo, la edad permitida para las misiones de las jóvenes tiene que ser más avanzada que la de los hombres, y en la fracción católica la participación en el carnaval presenta restricciones hacia las mujeres. Más allá de las instituciones familiares y religiosas, en su tiempo libre a los jóvenes les gusta:

(M) Pasar momentos con las personas que más nos agraden, con las personas que más nos hagan sonreír, con las personas que siempre estén ahí para ti, que tiendan a olvidar todo, que si van a jugar futbol se la pasen bien. (H) También salir con los amigos, tomar cervezas, unos cigarros, es otra manera de divertirse. (H) Yo individualmente me gusta escribir canciones, me gusta estar con mi guitarra, que afloren mis sentimientos, yo mismo me saco una sonrisa con mi trabajo hecho, eso es para mí divertirme individualmente. (M) En grupo, pues, salgo con mis amigos, me voy a pasear, vamos a comprar cosas, y ya individualmente me gusta dibujar vestidos y esas cosas, intercalar colores. $(\mathrm{H})$ Para mí es ir con los cuates, es ir a echar la reta. (M) Es que hay muchas maneras de divertirse, depende con qué persona o qué personalidad. En grupal... sí, te diviertes; individualmente, más que nada estar con tu familia, reflexionar escuchando música u otro tipo de diversión (grupo focal l, mayo de 2017).
Entre las prácticas relacionadas con el gusto juvenil se encuentran las vinculadas con las bandas y los bailes sonideros, que se asocian con la dinámica migratoria En Atexcac, la experiencia migratoria generó en la población una serie de capitales, entre ellos el incremento del capital de tipo social, que permitió a través de redes regionales la migración laboral a Filadelfia hacia finales de la década de 1990. A partir del año 2000, momento histórico en que algunas familias de Atexcac mejoraron sustancialmente su condición económica y material de vida, y que se consolida una dinámica de retorno migratorio, aparecieron las bandas juveniles y las prácticas del baile sonidero.

Así, el gusto por el baile sonidero y las bandas - estas últimas se asemejan al modelo de banda juvenil presente en el Estado de México estudiado por Feixa (1999), y que también es reproducido en Filadelfia, aunado a una serie de intereses e inquietudes juveniles vinculadas al modelo de juventud occidental - impulsa la aparición del sistema simbólico juvenil de Atexcac.

En cierta medida, lo que motivó la inserción y permite la reproducción de las prácticas vinculadas a las bandas fueron las tensiones territoriales entre los jóvenes y los adultos de Atexcac. Tales tensiones se deben a que los jóvenes contemporáneos entraron en contradicción con el sistema social tradicional de la localidad.

Las principales tensiones se experimentan desde el cuerpo, las identidades y las apuestas de vida individuales y colectivas. Los jóvenes son cuestionados por ver en la migración un nuevo proyecto de vida; por su vestimenta y por su ejercicio de la sexualidad; por su identificación con culturas juveniles urbanas que los inspiran para afirmar su identidad ayuuk y su pertenencia comunitaria, pero también los llevan a crear nuevas identidades juveniles inéditas en sus pueblos (Aquino y Contreras, 2016:473).

De la misma forma, en Atexcac, las tensiones entre jóvenes y adultos son concebidas como parte de la migración, aunque también pueden tener su origen en los movimientos religiosos - evangélicos y 
mormones-, que proporcionan a la juventud nuevos esquemas de interacción e intereses socioculturales. Además, abona a estas tensiones la consolidación de la escolarización, debido a que las disposiciones socioculturales que el Estado provee a los jóvenes a través de la escuela no son coherentes con la dinámica sociocultural de estos espacios, de tal manera que: "l) los contenidos educativos hacían alusión a realidades diferentes de las rurales; 2) los discursos científicos de la escuela polemizaban con los discursos tradicionales sobre el cuerpo, la familia y lo social" (Pacheco, 2013:131).

La lógica juvenil de estos elementos impone otro referente liminal de juventud vinculado a la cultura occidental, de tal manera que estas prácticas juveniles no estaban presentes en la dinámica sociocultural local de la generación anterior, lo que ha conducido a que los jóvenes contemporáneos gestionen un lugar que no estaba presente en el espacio social de Atexcac y demanden participar activamente en la dinámica territorial local.

En resumen, destacan dos cuestiones relevantes. Por un lado, que las prácticas juveniles son importadas de los lugares de destino laboral, son reproducidas por una fracción de hombres y mujeres jóvenes de la localidad y son resignificadas de acuerdo con sus intereses desde su manifestación a principios del siglo XXI. Por otro lado, que los subcampos de estas prácticas surgen por las tensiones territoriales entre los adultos y una nueva clase juvenil debido a que los jóvenes no encontraban un lugar propio en el espacio social de la comunidad. Esta situación obedeció a la ausencia de la figura del agente social joven contemporáneo en el sistema local $y$, por ende, a la ausencia de un lugar propio para ellos.

\section{Campos de inversión simbólica}

Algunas prácticas de los jóvenes están asociadas con las unidades domésticas de las que provienen, y de esta manera se integran a las diversas actividades que dispone la familia. En este sentido, la juventud de Atexcac, de acuerdo con su filiación religiosa — católica, mormona o evangélica-, cumple con los deberes que dispone la religión de la familia. Entre las prácticas católicas se encuentran la contribución a la organización de las celebraciones del sistema de cargos; en el caso de los mormones, asisten a reuniones especiales locales y regionales de la Iglesia, y se integran a las misiones - los hombres a partir de los 18 años y las mujeres a partir de los 21 - En el caso de los evangélicos, también ofrecen la opción de participar en reuniones locales y regionales.

Alinterior de estos sistemas, los jóvenes interesados en las prácticas compiten por los recursos que proveen, como el capital simbólico y social, que consiste en, por un lado, el reconocimiento del sector adulto y, por otro, en tener acceso a las redes sociales que proporciona cada grupo religioso. Los recursos en disputa de estos sistemas los disfruta la juventud a mediano y largo plazos, y los jóvenes interesados compiten por ellos a lo largo de su trayectoria de vida, cumpliendo con sus deberes y participando en las actividades comunes para obtenerlos.

Las prácticas asociadas a las religiones y a las obligaciones comunitarias se caracterizan por estar dominadas por las personas adultas, quienes son las que sacan mayor provecho de ellas. Por ejemplo, el prestigio que proporciona ser mayordomo en el sistema de cargos se deposita en los jefes de familia, aunque los jóvenes también contribuyan. En el caso de las redes sociales que genera la pertenencia a uno u otro grupo religioso, también son capitalizadas por el sector adulto, regularmente por los jefes de familia o por las personas formalmente encargadas de proveer bienestar social a su unidad doméstica; los jóvenes se benefician regularmente cuando conforman una familia o cuando dejan de considerarse jóvenes. En el ámbito cívico, los jefes de familia, generalmente hombres — con algunas excepciones - forman parte de las asambleas ejidales y comunitarias, con lo que solo ellos tienen voz y voto en las decisiones de la localidad, de manera que los jóvenes no participan en las decisiones principales de la comunidad. En tal sentido, cuando en un grupo focal se preguntó a un joven sobre su opinión en las asambleas locales, comentó:

Es que luego, muchas veces las palabras o lo que les dicen a los adultos, lo toman como una ofensa, porque 
yo he escuchado que dan su punto de vista o dan su opinión y las personas adultas como que se ofenden. Los adultos dicen cosas como: "Porque tú me gritas", "iEso te enseñaron en la escuela?", "Vas a la escuela a que te enseñen a desenvolverte no a criticar a otras personas". Pero si estás dando un punto de vista ipor qué lo toman a ofensa? Como que no tiene sentido (grupo focal l, mayo de 2017).

Estos ejemplos permiten determinar la posición marginal que tienen los jóvenes en las diferentes prácticas que los padres disponen para la juventud. En consecuencia, esta posición marginal se considera un factor que contribuyó al surgimiento y consolidación de las prácticas juveniles que son independientes y propias de este sector.

Entrevistador: ¿Y hasta cuándo podrían tener ustedes una voz dentro de la comunidad?

(H) Pues de hecho, de 30 para arriba, de veintitantos para arriba. (M) Cuando ya tienen obligaciones y ya pagas. (H) Desde el momento en que empiezas a pagar... (M) Así te van tomando en cuenta, porque si no... todavía eres parte de la familia de tu papá, y tu papá es el que va a hablar, no tú (grupo focal l, mayo de 2017).

Esta tensión es visible en muchos jóvenes de la localidad de Atexcac, tanto entre quienes están de acuerdo con la continuidad de la estructura sociocultural y demandan su integración en la toma de decisiones locales, como entre quienes han roto con la dinámica sociocultural y buscan nuevas modalidades de pertenencia. Aquellos que buscan estas nuevas formas de pertenencia son principalmente jóvenes ligados a las dinámicas de movilidad laboral y de migración. Se distingue, así, que la experiencia migratoria es un agente de transformación que los jóvenes de la localidad dirigen hacia la reestructuración de su entorno social. En los lugares de destino migratorio encuentran nuevas formas de ser que están ligadas a su condición etaria, entre las que destaca la dinámica de las bandas. Sin embargo, si bien la migración favorece los recursos estructurales para la aparición de estas bandas, las condiciones locales y la ideología juvenil contemporánea — apoyada de las redes sociales migratorias, las disposiciones escolares y los mass media - prevén su consolidación y reproducción particular en Atexcac.

Al margen de los sistemas de organización religiosos y civiles, los jóvenes se desenvuelven en otras prácticas independientes del sector adulto. Destacan en la comunidad, por ejemplo, las prácticas del futbol, los bailes sonideros y las bandas. En el caso del futbol, se encuentra un grupo juvenil constituido particularmente por hombres que se disputan el reconocimiento social de los jóvenes que socializan en los espacios deportivos y que en las tardes de cualquier día se pueden observar en las canchas de la localidad.

\section{Bandas y bailes sonideros}

Las prácticas juveniles de las bandas y los bailes sonideros tienen diferente origen. Mientras que las bandas se relacionan con los estilos que jóvenes de la región reproducen de Filadelfia, Estados Unidos, los bailes sonideros se asocian a un movimiento regional del valle poblano. Sin embargo, estas estructuras son readaptadas en la localidad y se han consolidado por efecto de las necesidades del sector juvenil contemporáneo de Atexcac, tanto por los jóvenes que demandan ser incluidos en la dinámica sociocultural de la localidad, como por los que se ven motivados por las disposiciones juveniles occidentales.

En cuanto a la procedencia de la organización de bandas, los jóvenes comentaron:

(H) No, pues casi no se veía, se empezó por el 2000 en adelante, por los que se iban a Estados Unidos y regresaban y traían diferente visión. (M) Se conforman a partir de los que no se llevan bien, y dicen "no, pues yo mejor me llevo a mis amigos para allá"; bueno, yo digo que por eso se arman. Sí, porque yo estaba niño cuando empezaron, unos valedores grandes, señores que regresaron ya con un tatuaje o algo. (H) Algunos quedan, pero ya no lo hacen (grupo focal l, mayo de 2017) 
El sistema de bandas de Atexcac se distingue por su similitud con la estructura sociocultural local; por ejemplo, el sistema basado en la celebración del aniversario de las bandas que recompensa con capital simbólico a los jóvenes se asemeja al de las mayordomías del sistema de cargos, que cede esta misma recompensa a los adultos.

El baile sonidero se incorpora como una de las actividades regulares y representativas del sistema de bandas, aunque también son organizados por los jóvenes al margen de aquellas. El sentido del baile sonidero en los jóvenes de esta localidad se parece al propuesto por Salazar, para quien: "La música del sonidero será cada vez menos una música escuchada por pasatiempo, y se volverá cada vez más en un 'estilo de vida', una forma de pensar y de confrontación con la realidad, una forma de articular emociones en colectivo" (Salazar, 2018:61).

En la incorporación de los bailes sonideros a la dinámica juvenil local se considera la vecindad territorial que hay entre Huejotzingo y la ciudad de Puebla. En igual forma, de acuerdo con González (2003), el "medio sonidero" aparece desde la década de 1980, y en la actualidad es representativo y exponencial de Cholula, Puebla, municipio territorialmente próximo a Huejotzingo.

Las prácticas son analizadas como subcampos. Para la construcción de los subcampos se tomó en cuenta el interés que los jóvenes tienen hacia las prácticas. Con el análisis del habitus juvenil, que valora la cotidianidad y los gustos de este sector, se encontró que su interés está dirigido hacia diversas prácticas, entre ellas los bailes sonideros y bandas. Se consideran subcampo porque las prácticas de análisis forman parte de un gran grupo de prácticas locales que están orientadas hacia la obtención de bienes como el estatus y el reconocimiento social. Este bien se comprende como una recompensa, que se denomina capital simbólico.

La relación entre subcampo y capital se da por la consideración que comprende las prácticas como parte de un sistema estructurado de recompensas en el que los agentes sociales mueven sus recursos para obtenerlas. De esta manera, los jóvenes de Atexcac, para alcanzar estatus o capital simbólico, tienen que movilizar diferentes recursos a fin de lograr ser reconocidos socialmente. Cabe señalar que el subcampo juvenil simbólico de Atexcac, además de generar estatus entre jóvenes, les ofrece la oportunidad de mostrarse ante el mundo adulto y de demostrar que son capaces de organizarse y hacer propuestas.

Como se observa en la Figura 1, los subcampos tienen independencia relativa de lo que sucede en el espacio social de Atexcac, es decir, que aunque en el subcampo se juega con y por los sujetos jóvenes, tiene reglas propias y una especie de capital singular; muchos de los recursos utilizados provienen de las unidades domésticas de origen de los jóvenes. Por ejemplo, en los bailes sonideros es particularmente visible el capital económico, que es esencial para la contratación del sonido y la adquisición de bebidas.

En la Figura 1, el cuadro pequeño y bicolor representa el campo, y cada lado del cuadro un subcampo que contempla cada grupo de prácticas. Cada subcampo se ordena de acuerdo con los instrumentos de reproducción o grupos de prácticas que se erigen por la competencia hacia el capital simbólico. Las esferas al interior de cada subcampo representan la posición que los agentes tienen. Finalmente, las posiciones que los agentes sociales ocupan en cada subcampo están condicionadas por el volumen de capital (ver Figura 1).

\section{Subcampo bailes sonideros}

El subcampo de los bailes sonideros está constituido por las prácticas en su organización y por la participación directa. Los bailes son organizados tanto por los jóvenes pertenecientes a una banda, como por otros que se mueven al margen de estas. En el caso de las bandas, los bailes se organizan con motivo de su aniversario, y en el caso de los jóvenes no vinculados con bandas, los organizan para festejar, por ejemplo, cumpleaños o por el interés de mostrarse para ser reconocidos. Los que están al margen de las bandas organizan bailes entre grupos de amigos, y para ello se reparten tareas y los montos de cooperación. 
En todos los casos llevan a cabo sistemas de organización y cooperación que se asemejan a las prácticas del sistema de cargos de la localidad; por ejemplo, en la mayordomía el festejado y sus allegados proporcionan recursos para llevar a cabo una celebración sobresaliente que contribuya a favorecer el prestigio de un agente social, que puede ser una banda, un grupo de amigos o un individuo. La similitud se observa en la forma de organización de los bailes sonideros, que también se basa en un sistema de ayuda comunitario, aunque en este caso amical.

Según el desenvolvimiento y la actuación en el campo, los jóvenes se enfilan a obtener el capital simbólico; por efecto de reconversión, las recompensas van desde conseguir una pareja sentimental, hasta ser reconocido por las aptitudes que tienen para bailar o por las habilidades para pelear a golpes. En este sentido, cuando se les preguntó sobre lo que les gusta de los bailes, contestaron: "Bailar; bailar más que nada; los trancazos; hay veces que se pasan a pelear o así, pero para mí me gusta más ir a bailar; más que nada las chicas" (grupo focal 1, mayo de 2017).

Para la obtención de capital simbólico en los bailes sonideros, la organización es más efectiva que la participación o desenvolvimiento en estos, porque los organizadores obtienen respeto y reconocimiento de acuerdo con la calidad de su fiesta, donde los parámetros de éxito dependen de la contratación de un sonidero reconocido, con un equipo de sonido excesivo y ruidoso, y la disposición de bebidas embriagantes en gran cantidad para los participantes. Para lograr una fiesta con estas particularidades, es vital la inversión de capital económico, que proviene principalmente de dos fuentes, de la unidad doméstica y de los recursos propios de la juventud.

En la organización de las fiestas de las bandas, los recursos provienen también de los propios jóvenes y de sus unidades domésticas de procedencia. Es importante mencionar que las bandas están conformadas en buen número por jóvenes con una condición sociocultural particular asociada a la migración y a la movilidad laboral, que generan una condición particular de independencia juvenil. Ello se debe a que es común que los hijos de migrantes se queden en la comunidad bajo resguardo de los abuelos o algún pariente, aunque algunos también viven solos y se mantienen con las remesas que los padres les envían. Sin la vigilancia de los padres, los jóvenes encuentran más libertad para moverse en las prácticas de las bandas, y con el capital económico que reciben tienen la solvencia monetaria para desenvolverse en esta dinámica juvenil y para organizar los bailes sonideros.

El volumen del capital de los jóvenes, que está vinculado con el de las unidades familiares, influye en la disputa por el estatus social del subcampo de los bailes sonideros. Así, pueden encontrarse dos subclases socioculturales de jóvenes en el subcampo de los bailes sonideros. La diferencia se encuentra entre quienes los organizan y quienes solo participan en ellos. Regularmente, los que organizan las fiestas son los que tienen mayor volumen de capital, particularmente de tipo económico, por lo que cuentan con más posibilidades de hacerse del capital simbólico. Por el contrario, los jóvenes que solo participan tienen la posibilidad de obtener ciertas ganancias, pero no el capital principal en disputa.

Pese a que los agentes y organizadores pueden estar en condiciones económicas diferentes, y quienes organizan regularmente las fiestas cuentan por logeneral con mayor capital económico, todos los participantes - aún aquellos que provienen de unidades domésticas con menor volumen de capital — tienen la posibilidad de organizar una fiesta y obtener el capital en disputa. Esto se debe a que los sistemas de organización y cooperación derivan de los sistemas tradicionales locales, lo que permite de manera grupal organizar un baile sonidero. La condición del uso de estos sistemas es el capital social, lo cual no es un problema para cualquier joven de la localidad debido a que las redes juveniles son en gran medida de tipo parental.

Así, pese a que todos los jóvenes tienen la posibilidad de acceder al capital simbólico generado por los bailes, son quienes poseen mayor volumen de capital los que se encuentran siempre más cerca de obtenerlo.

En las prácticas de los bailes sonideros se observan diferentes estrategias que son extraídas de la propia 
localidad, es decir, que los jóvenes han reestructurado las prácticas tradicionales adoptando las disposiciones socioculturales propias de la localidad. Además, han configurado sus prácticas de tal manera que intentan cubrir las necesidades y los intereses de los jóvenes de la localidad, en este caso lograr capital simbólico, que es un bien que les permite ser conocidos y reconocidos y disponer de un lugar en el espacio social, algo que no poseen quienes se mueven al margen de las instituciones y quienes se identifican con otras dinámicas y estilos juveniles que no son propiamente del lugar.

\section{Subcampo bandas}

De igual manera, las prácticas que llevan a cabo las bandas en Atexcac les brindan un lugar en el espacio social del lugar. En estas agrupaciones participan jóvenes de entre 14 y 25 años, aunque es menos común encontrar mayores de 18 años. Están integradas por jóvenes de diferentes características: algunos son estudiantes que cursan secundaria o preparatoria; otros han truncado sus estudios y laboran; participan jóvenes de las religiones católica, mormona y evangélica; unos son hijos de migrantes o de campesinos de la localidad; algunos trabajan, mientras otros no estudian ni trabajan; participan hombres y mujeres.

Las bandas se constituyen de acuerdo con una división territorial, que deriva, en primer lugar, de la división tradicional de Atexcac, donde existe una distinción entre el "barrio de arriba" y el "barrio de abajo" y, en segundo lugar, de la colonia en la que surgen. La cantidad de integrantes de las bandas oscila entre los quince y treinta miembros. En total hay alrededor de ocho bandas, una de ellas reconocida por estar constituida solo por mujeres. Sobre las bandas que existen actualmente comentaron:

Arriba están los Morris, los Sur 13, los Quinto Sol; hay una bandita de puros chamacos de como 15 y 17 años que se decían los KDC; y los de abajo los AR [Amantes de la Reforma], los Quicks y los Callejeros; arriba los Infieles Forever; de las mujeres, hay una bandita que se llama las Crazy (grupo focal 1, mayo de 2017).
Entre las prácticas que llevan a cabo las bandas destacan los bailes sonideros, que organizan con motivo de la celebración de su aniversario. "Pues aquí se organizan varios jóvenes, y dicen 'vamos a hacer una fiesta'; son como grupos que forman, y cada año ellos tienen su aniversario; se organizan, cooperan" (grupo focal 2, mayo de 2017).

Las reuniones de los integrantes de las bandas son una práctica común. En ellas los jóvenes interactúan y dialogan sobre intereses particulares, como las bandas, las fiestas, los conflictos o sus experiencias como parte de la agrupación. En estas reuniones por lo regular hacen uso de algún estupefaciente y no tienen horario; desde medio día se pueden ver agrupaciones de jóvenes en alguna esquina del pueblo, en la fachada de una casa o en alguna tienda.

También los conflictos son propios de las bandas, los cuales regularmente llegan a ser violentos. Por lo común son premeditados, propios de los hombres, y surgen cuando dos bandas se encuentran deambulando en las calles o en las fiestas.
Van preparados, más que nada te marcan, ya llegan, ya es hora. Pero ya cuando dicen ya es hora es porque ya... ya empezaron con todo; hay unos chavos que ya no quieren pelear a lo que se dice "mano limpia", ya sacan los cuchillos, bats, las cadenas, navajas, lo que encuentran; hasta la leña de los vecinos (grupo focal 1, mayo de 2017).

Estas prácticas, que incluimos como parte del subcampo bandas, dotan de capital simbólico a los jóvenes, tanto a los que participan en las bandas como a los que no. Entre los jóvenes, aunque no pertenezcan a ninguna banda, son bien conocidos los nombres o seudónimos de los integrantes de las bandas más famosos.

Para obtener reconocimiento social y respeto entre los integrantes de la banda es necesario ser partícipe de las prácticas comunes como: estar presente en las reuniones, apoyar en la organización de los aniversarios, destacarse durante las fiestas y exhibir habilidades para liarse a golpes. Este último caso no 
se presenta en las bandas de mujeres, quienes no ven necesaria la violencia.

A diferencia de lo que sucede en el caso de los bailes, el volumen de capital en el subcampo bandas es dominado por el capital simbólico que se obtiene al interior de cada banda. Asimismo, la apertura del sistema de bandas contribuye a que otros agentes sociales se interesen por su dinámica y se integren a ellas.

En general, las bandas ofrecen a la juventud capitales simbólicos que se contraponen a las prácticas dominantes en la localidad, donde el sector adulto es el privilegiado y domina sobre los recursos disponibles. Así, los sistemas mencionados proporcionan un lugar a los jóvenes que la tradición no concede debido a las condiciones sociohistóricas locales, que desconocen la figura del joven contemporáneo.

\section{Conclusiones}

En Atexcac, la dinámica socioeconómica basada en la migración, junto con otros factores como la escolarización y la diversificación religiosa, han generado procesos de transformación social. Las contradicciones presentes entre la dinámica actual de la sociedad de Atexcac y las reglas socio-tradicionales han generado una brecha de desigualdad entre los agentes sociales jóvenes y los adultos de la localidad. Estas contradicciones tienen que ver con la emergencia de una clase social juvenil a principios del siglo XXI $y$, derivado de ello, con el surgimiento de una serie de demandas tácitas y explícitas entre las que destaca la necesidad de un lugar, de adquirir voz.

Si bien anteriormente las personas pasaban a ser adultas entre los 12 y 15 años y comenzaban a participar en actividades comunitarias - lo que implicaba tomar parte en las decisiones locales-, en la actualidad la juventud prolonga su incorporación a la dinámica social adulta, lo que ha motivado a este sector a buscar por otros medios un lugar en el espacio social de Atexcac. Así, los bailes sonideros y las bandas se han integrado como recursos juveniles que proporcionan a la juventud un lugar en la localidad.
Los bailes sonideros y las bandas son una respuesta a la necesidad contemporánea juvenil de ser y sentirse parte de la comunidad. Son prácticas que provienen de modelos y recursos de las sociedades de destino migratorio y laboral, pero que han sido reinterpretadas y adecuadas a sus disposiciones, necesidades e intereses, incluyendo simultáneamente algunos recursos organizativos del sistema tradicional local. Además, estas prácticas están orientadas a la obtención de un bien de reconocimiento, una cualidad marginal de los jóvenes en el espacio social de Atexcac, por lo que buscan legitimar sus prácticas más allá del sector juvenil.

Finalmente, es importante reconocer que no todos los jóvenes de la localidad están interesados en estas prácticas, que hay diferencias entre la actuación de hombres y mujeres - aunque comparten un interés común en el capital simbólico-, y que se presentan discrepancias de disposición e interés hacia las prácticas dependiendo de la filiación religiosa o del vínculo con las redes migratorias, de manera que los católicos y los jóvenes asociados a las dinámicas migratorias son los que presentan mayor disposición a la reproducción de estas prácticas.

\section{Referencias}

Aquino, Alejandra e Isis Contreras Pastrana (2016). "Comunidad, jóvenes y generación: disputando subjetividades en la Sierra Norte de Oaxaca." En Revista Latinoamericana de Ciencias Sociales, Niñez y Juventud, 14(1): 463-475. Disponible en: http:// revistalatinoamericanaumanizales.cinde.org.co

Bourdieu, Pierre (2011). Las estrategias de la reprodución social. Buenos Aires: Siglo XXI.

Bourdieu, Pierre (2013). El oficio del sociólogo. Presupuestos epistemológicos. México: Siglo XXI.

Bourdieu, Pierre (2015). El sentido social del gusto: elementos para una sociología de la cultura. México: Siglo XXI.

Feixa, Carles (1999). De jóvenes, bandas y tribus. Antropología de la Juventud. Barcelona: Ariel.

Feixa, Carles, Ariadna Fernández Planells y Mónica Figueras Maz (2012). “Generación Hashtag. Los 
movimientos juveniles en la era de la web social". En Revista Latinoamericana de Ciencias Sociales, Niñez y Juventud, 14(1), 107-120.

García Martínez, Ariel (2012). "Juventud indígena en el Totonacapan Veracruzano". En LiminaR. Estudios Sociales y Humanísticos, X(1),75-88. Disponible en: http:// liminar.cesmeca.mx/index.php/rl/article/view/38/0

González, Eduardo (2003). "Sonidero poblano". En Mirada Antropológica, 1, enero-julio, 93-116.

Gutiérrez, Alicia B. (2005). Las prácticas sociales. Una introducción a Pierre Bourdieu. Buenos Aires: Ferreyra Editor.

Hernández Carrera, Rafael (2014). "La investigación cualitativa a través de entrevistas: su análisis mediante la teoría fundamentada”. En Cuestiones Pedagógicas, 23, 187-210. Disponible en: http://institucional.us.es/ revistas/cuestiones/23/Mis_5.pdf
INEGI (Instituto Nacional de Geografía e Informática (2010). Censo de Poblacióny Vivienda 2010. México: INEGI. Disponible en: https://www.inegi.org.mx/programas/ ccpv/2010/default.html\#Microdatos (consultado el 15 de agosto de 2012).

Pacheco Ladrón de Guevara, Lourdes (2013). "Los últimos guardianes. Jóvenes rurales e indígenas". En Rosana Reguillo (coord.), Los jóvenes de México. México: FCE.

Salazar Lara, Flor Andrea (2018). Movimiento sonidero: construcción de socialidades y visualidades. Tesis de maestría, Universidad Autónoma del Estado de México, México.

Urteaga, Maritza (2008). "Jóvenes e indios en el México contemporáneo." En Revista Latinoamericana de Ciencias Sociales, Niñezy Juventud, 6(2), 667-708. Disponible en: http://www.redalyc.org/pdf/773/77360207.pdf

Figura 1. Campo simbólico juvenil de Atexcac

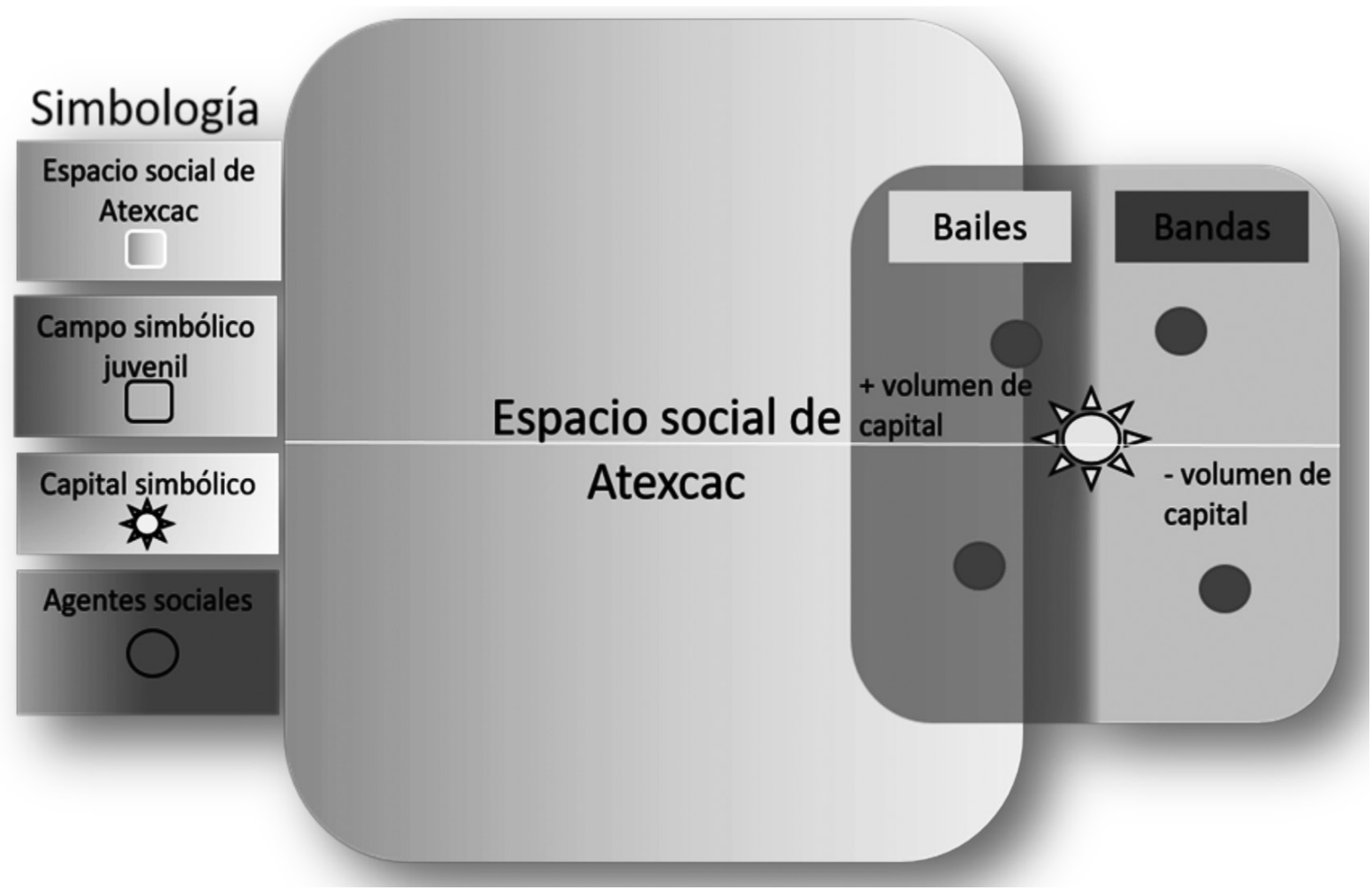

Fuente: elaboración propia, 2019. 